\title{
Phonon Softening and High-Pressure Low-Symmetry Phases of Cesium Iodide
}

\author{
Marco Buongiorno Nardelli and Stefano Baroni \\ Scuola Internazionale Superiore di Studî Avanzati (SISSA), Via Beirut 2/4, I-34014 Trieste, Italy \\ Paolo Giannozzi \\ Scuola Normale Superiore (SNS), Piazza dei Cavalieri 7, I-56100 Pisa, Italy \\ (Received 15 April 1992)
}

\begin{abstract}
The relative stability of various high-pressure phases of CsI is studied from first principles and analyzed using the Landau theory of phase transitions. We demonstrate that the cubic-to-orthorhombic transition recently observed to occur slightly below $20 \mathrm{GPa}$ is driven by the softening of an acoustic phonon at the $M$ point of the Brillouin zone. The coupling between this mode and anisotropic strain makes the transition slightly first order (with a volume variation of the order of $0.1 \%$ ), and stabilizes the experimentally observed orthorhombic phase with respect to other competing symmetry-allowed structures.
\end{abstract}

PACS numbers: $62.50 .+\mathrm{p}$

The high-pressure properties of CsI are the subject of current experimental interest both in conjunction with band-overlap metallization and also because this cubic salt undergoes a rather unexpected transition to a lowsymmetry phase at an applied pressure of a few tens of GPa [1-3]. Until recently, the commonly accepted picture was that a cubic-to-tetragonal martensitic transition would occur at a pressure of about $40 \mathrm{GPa}$. A further transition from the tetragonal to an orthorhombic phase at $56 \mathrm{GPa}$ has been reported in Ref. [2], but this finding has not been confirmed by subsequent experimental $[1,3]$ nor theoretical [4-7] work. In particular, though the cubic-to-tetragonal transition has been theoretically predicted by both semiempirical [4] and first-principles $[5,6]$ calculations, no evidence of the further orthorhombic distortion has been found by either methods [7].

Recent experiments [8,9] suggest that CsI undergoes a continuous transition from the cubic (B2) to an hcp structure, passing through an orthorhombic phase, $C_{2 v}^{1}$, which is, however, different from the previously proposed structure [2], $D_{2 h}^{1}$. In this paper we study the relative stability of various phases of CsI at high pressure (cubic, tetragonal, and the newly proposed orthorhombic structure). We identify the amplitude of a sixfold-degenerate phonon mode $\left(\mathrm{M}_{5}^{-}\right)$as the relevant order parameter of the transition in the Landau sense. The frequency of this phonon is found to vanish at a pressure of $\approx 23 \mathrm{GPa}$, which is well below the transition from the cubic to the tetragonal phase. Neglecting the coupling between the soft mode and anisotropic macroscopic strain, we find that the transition would be second order from the cubic to a tetrahedral $\left(T^{5}\right)$ phase; the coupling with macroscopic strain stabilizes the orthorhombic structure, making the transition first order, with a very small volume change $(\approx 0.1 \%)$ and a transition pressure $(\approx 21 \mathrm{GPa})$ slightly below the softening pressure of the $\mathrm{M}_{5}^{-}$phonon. We also find that the orthorhombic structure is always favored with respect to the tetragonal structure, up to pressures of $60 \mathrm{GPa}$.

The cubic-to-tetragonal transition reported in Refs. [1-3] was originally thought to be second order. Group- theoretical considerations show that this transition must be first order [10], and a discontinuity in the order parameter, $c / a$, at the transition was actually found in both semiempirical [4] and first-principles [5,6] calculations. Even so, this transition is driven by a dramatic softening of the shear constant, $c_{s}=\frac{1}{2}\left(c_{11}-c_{12}\right)$, which in fact vanishes at a volume slightly below the transition [4-6]. The shear constant is also proportional to the square of the sound velocity along the (110) direction for vibrations polarized along ( $1 \overline{1} 0)$. This observation suggests that the softening of the shear constant could be associated with the softening of a transverse phonon along the (110) direction. In fact, the gliding of one of the (110) planes-which was indicated in Ref. [8] as characterizing the low-symmetry phase of CsI-represents a lattice distortion which is very similar to that due to a doubly degenerate acoustic phonon mode at the $M$ point of the Brillouin zone (BZ), $\mathrm{M}_{5}^{-}$.

In order to clarify the nature of the transition, we have performed extensive density-functional-theory (DFT) calculations of the static and vibrational properties of CsI at high pressure, using the local-density approximation (LDA), norm-conserving pseudopotentials, and large plane-wave basis sets. The computational details are similar to those described in Ref. [6], with the only difference that we have now used a somewhat larger kinetic-energy cutoff $(25 \mathrm{Ry})$ : The resulting values of the equilibrium lattice constant and bulk modulus are $4.44 \AA$ and $15 \mathrm{GPa}$, respectively. The vibrational properties have been calculated using the density-functional perturbation theory described in Ref. [11]. In Table I some phonon frequencies calculated at the equilibrium volume are compared with experimental data. The agreement is quite satisfactory, giving confidence in the predictive power of our calculations.

In Fig. 1 we display the ionic displacements along the normal coordinates of the $M_{5}^{-}$acoustic phonon [14], together with the dependence of the corresponding frequency upon molar volume. The displacement pattern is similar to but different from that proposed in Ref. [8] (the magnitude of the cationic and anionic displacements are 
TABLE I. Comparison between calculated and experimentally observed phonon frequencies of CsI at zero pressure, at the $\Gamma$ and $M$ points of the Brillouin zone. Units are THz. The uncertainty on the last value in the experimental data row is indicated in parentheses.

\begin{tabular}{lllllll}
\hline \hline & $\omega_{\mathrm{TO}}(\Gamma)$ & $\omega_{\mathrm{LO}}(\Gamma)$ & $\omega_{\mathrm{TA}}(M)$ & $\omega_{\mathrm{LA}}(M)$ & $\omega_{\mathrm{TO}}(M)$ & $\omega_{\mathrm{LO}}(M)$ \\
\hline Theory & 2.09 & 2.85 & 1.17 & 1.30 & 1.48 & 2.35 \\
Expt. & $1.91(5)^{\mathrm{a}}$ & $2.74(1)^{\mathrm{b}}$ & $1.29(6)^{\mathrm{a}}$ & $1.34(5)^{\mathrm{a}}$ & $\cdots$ & $2.26(5)^{\mathrm{a}}$ \\
\hline \hline
\end{tabular}

${ }^{a}$ Experimental data from Ref. [12]. $\quad{ }^{b}$ Experimental data from Ref. [13].

different here, their ratio depending on the actual dynamical matrix of the crystal). The $M_{5}^{-}$phonon softens at $v \equiv V / V_{0}=0.64$, corresponding to a pressure $P \approx 23 \mathrm{GPa}$, as a consequence of the incipient softening of the sound velocity of one of the transverse branches (see inset). The vanishing of the $M_{5}^{-}$frequency signals the onset of a phase transi-

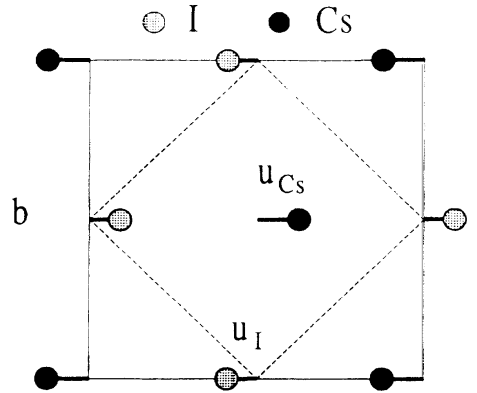

a

$P(\mathrm{GPa})$

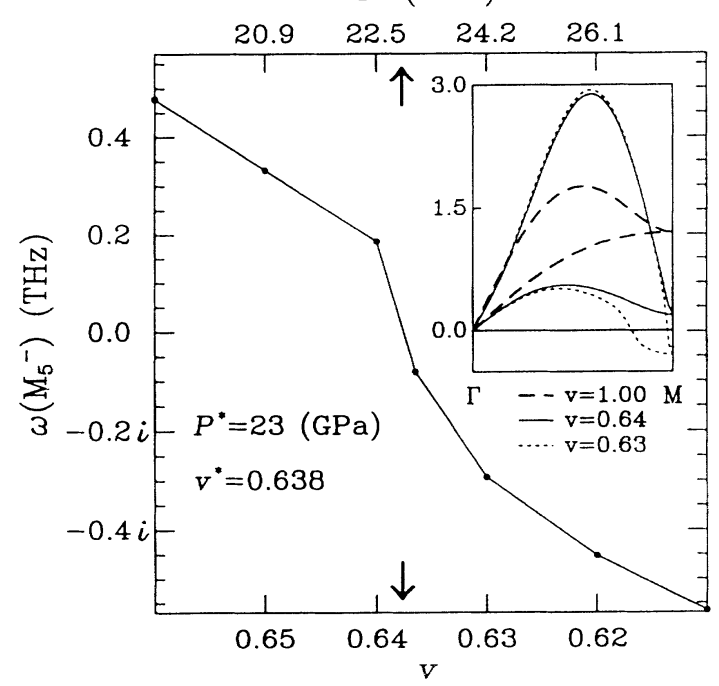

FIG. 1. Upper panel: Atomic displacements corresponding to an $M_{5}^{-}$acoustic phonon, viewed on the $(001)$ plane. The magnitude of iodine displacements $\mathbf{u}_{\mathrm{I}}$ with respect to cesium displacements $\mathbf{u}_{\mathrm{Cs}}$ is exaggerated for clarity. The solid (dashed) line indicates the unit cell of the orthorhombic (cubic) structure. Iodine atoms lie on a plane shifted along the $z$ direction by $c / 2$ with respect to the cesium plane. In the cubic structure one has $a=b=\sqrt{2} c$. Lower panel: Frequency of the $M_{5}-$ phonon as a function of molar volume (lower scale, $v \equiv V / V_{0}$, where $V_{0}$ is the equilibrium volume) and of the pressure (upper scale). Arrows indicate the softening volume $\left(v^{*}\right)$ and pressure $\left(P^{*}\right)$. Inset: The lower portion of the acoustic dispersion along (110) at equilibrium volume (dashed line), and at volumes slightly above (solid line) and below (dotted line) the mode softening. tion, whose order parameter is the amplitude of the atomic displacements along the phonon normal coordinates [14]. The small group of the $M$ point-whose coordinates are $\left(\frac{1}{2} \frac{1}{2} 0\right)-$ is $D_{4 h}$ and its star is made of three equivalent points. The $\mathrm{M}_{5}^{-}$mode transforms according to the doubly degenerate $\Gamma_{5}^{-}$irreducible representation of $D_{4 h}$, so that the order parameter associated with this mode is six dimensional. According to the Landau theory [15], the transition can be second order if no third-order cubic invariants can be constructed with the components of the order parameter. This is manifestly the case here, since the $\Gamma_{5}^{-}$representation is odd. Assuming that the sixth-and higher-order terms in the development of the free energy in powers of the order parameter are positive in the neighborhood of the high-symmetry phase, the transition is then first or second order, according to whether the fourth-order invariants are negative or positive, respectively. A classification of fourth- and sixthorder invariants for six-dimensional representations of space groups is given in Ref. [16]. In our case, there are five fourth-order invariants whose coefficients have been determined in the particular case $V / V_{0}=0.58$ by fitting their values with DFT calculations done for a few independent lattice distortions. All these invariants turn out to be positive, thus indicating that the transition would be second order. Group-theoretical considerations considerably restrict the number of possible low-symmetry phases. According to Ref. [16], there are seven distorted crystal structures of "maximal isotropy" (each structure corresponds to a different "direction" in the six-dimensional configuration space of the order parameter). In the present case, an analysis of the crystal energy reveals that below the transition the only energy minimum occurs along the so-called " $P 11$ " direction [16] which corresponds to the simultaneous excitations of all the six degenerate soft modes. The space group of the distorted phase is $T^{5}$, whose tetrahedral symmetry cannot be reconciled with the observed $x$-ray diffraction patterns [8].

The above considerations hold in the hypothesis that the strain state of the crystal is constant across the transition, except for the isotropic compression due to the application of a hydrostatic pressure. We know, however, that the softening of the $M_{5}{ }^{-}$phonon mode is closely related to the softening of the shear constant of the crystal, 
so that a strong coupling between the soft mode and macroscopic strain (i.e., between zone-center and zone-border acoustic phonons) is to be expected. We consider next the expression of the Landau enthalpy of the crystal up to fourth order in the phonon displacements, including the coupling with the strain [17]. We find that the $P 11$ minimum previously found is unaffected by such a coupling, while four out of the other six maximal isotropy directional minima are slightly modified, still maintaining the saddle-point character they would have ignoring the phonon-strain coupling. This coupling changes instead the character of the minima along the other two maximal isotropy directions, $P 1$ and $P 2$, turning them from saddle points to true minima. The $P 1$ structure corresponds to the excitation of just one transverse acoustic phonon (see Fig. 1). When the coupling with strain is neglected, this structure has a much lower energy than that of the $P 2$ one which corresponds to the simultaneous excitations of two degenerate soft phonons at the same point of the BZ. The observed $x$-ray diffraction pattern from the lowsymmetry phase of CsI [8] is indeed compatible with the space group of the $P 1$ structure, $D_{2 h}^{5}$, which is furthermore geometrically simpler than the $P 2$ one. For these reasons, in the following we will concentrate on the $P 1$ distortion only.

Along the $P 1$ direction, the power expansion of the crystal enthalpy reads [17]

$$
\begin{aligned}
H\left(u, \epsilon_{1}, \epsilon_{2}\right)= & \frac{1}{2} k u^{2}+a u^{4}+\frac{1}{2} c_{s} \epsilon_{1}^{2}+\frac{1}{2} c_{44} \epsilon_{2}^{2} \\
& +\left(b_{1} \epsilon_{1}+b_{2} \epsilon_{2}\right) u^{2}+O\left(u^{6}\right),
\end{aligned}
$$

where $u$ is the amplitude of the phonon displacement along (1i0), $\epsilon_{1}=\frac{1}{2}\left(\epsilon_{x x}+\epsilon_{y y}-2 \epsilon_{z z}\right)$, and $\epsilon_{2}=\epsilon_{x y}, \epsilon_{i j}$ being the strain tensor. By eliminating $\epsilon_{1}$ and $\epsilon_{2}$ from Eq. (1) by the condition $\partial H / \partial \epsilon_{1}=\partial H / \partial \epsilon_{2}=0$, one obtains

$$
\begin{aligned}
& \epsilon_{1}=-\frac{b_{1} u^{2}}{c_{s}}, \epsilon_{2}=-\frac{b_{2} u^{2}}{c_{44}}, \\
& \tilde{H}(u)=\frac{1}{2} k u^{2}+\left(a-\frac{b_{1}^{2}}{2 c_{s}}-\frac{b_{2}^{2}}{2 c_{44}}\right) u^{4}+O\left(u^{6}\right) .
\end{aligned}
$$

Equation (3) shows that the coupling between the soft phonon and macroscopic strain renormalizes the fourthorder coefficient, making it large and negative, whenever $c_{s}$ or $c_{44}$ are small enough. As a result of the ongoing softening of the shear constant $c_{s}$, we do find that at the softening pressure the fourth-order coefficient is negative, and we conclude that the transition must then be first order, occurring at a somewhat lower pressure. A thorough study of the transition could be performed by considering the expansion of the crystal energy up to sixth order in the order parameter, and by fitting the relevant coefficients with first-principles calculations, as was done when the coupling to the strain was neglected. We have preferred instead to perform straight energy minimizations with respect to $u$ and $\sigma$ along the $P 1$ line, in correspondence to different volumes, so as to directly obtain the equation of state of the crystal in the low-

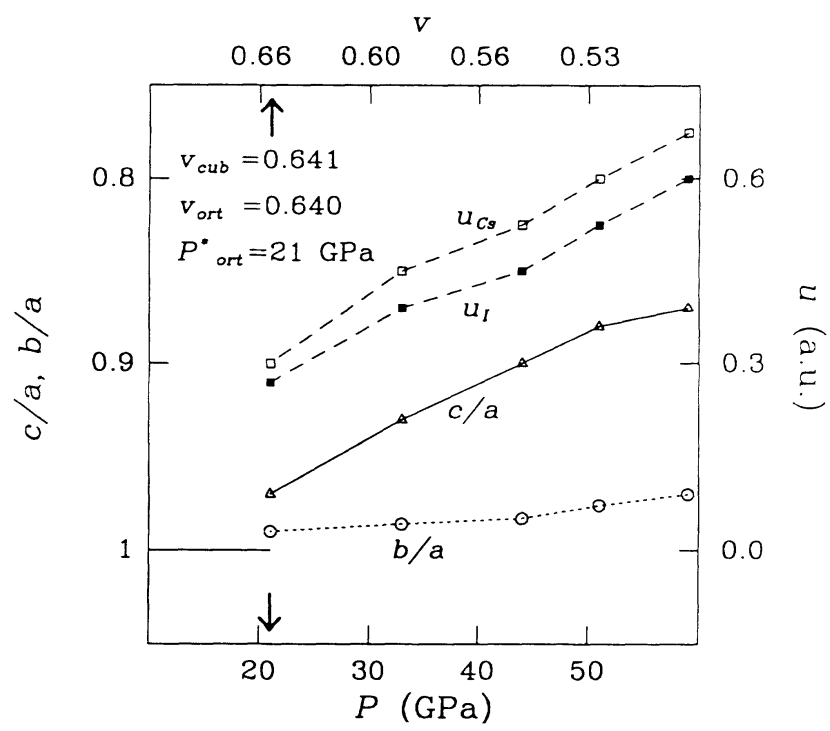

FIG. 2. Structural parameters of the orthorhombic phase (see Fig. 1) of CsI as functions of different values of pressure and volume. Arrows indicate the transition pressure and volume. The volume discontinuity at the transition is not visible on this scale. $P_{\text {ort }}^{*}$ indicates the transition pressure, while $v_{\text {cub }}$ and $v_{\text {ort }}$ are the volumes of the high- and low-symmetry phases at the transition, respectively.

symmetry phase. The Maxwell construction gives then a transition pressure of $\approx 21 \mathrm{GPa}$, corresponding to a volume in the cubic phase $V / V_{0} \approx 0.64$, which is actually slightly larger than the softening volume of the $\mathrm{M}_{5}^{-}$phonon.

In Fig. 2 we report the structural parameters of the orthorhombic phase, calculated for different values of the pressure and volume. All the structural parameters, including volume, are discontinuous at the transition pres-

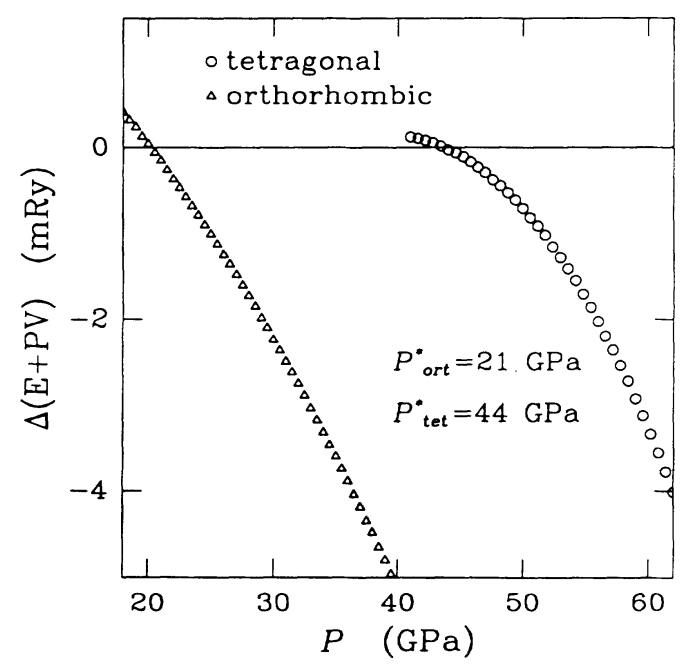

FIG. 3. Enthalpies of the orthorhombic and tetragonal phases, relative to the enthalpy of the cubic phase, as functions of the applied pressure. $P_{\text {ort }}^{*}$ and $P_{\text {tet }}^{*}$ are the transition pressures from the cubic to the orthorhombic and tetragonal phases, respectively. 


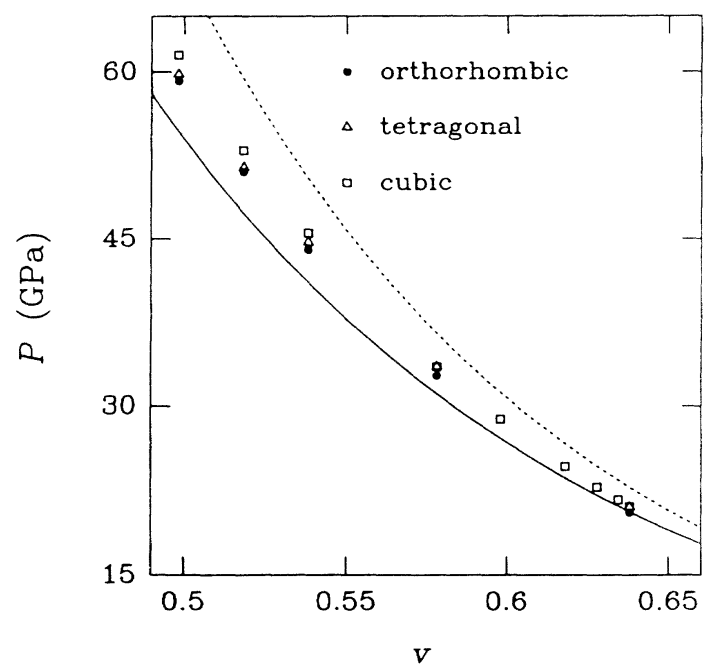

FIG. 4. Pressure vs volume data calculated in the present work for different phases of CsI, compared to previous equations of state (dashed line from Ref. [2] and solid line from Ref. [8]), fitted to experimental data.

sure as a consequence of its first-order character. The volume discontinuity is very small $(0.1 \%)$ and it is not visible on the scale of the figure. Note also the different magnitudes of the cesium and iodine displacements, and the very weak dependence of $b / a$ upon applied pressure. The different behavior of $c / a-1$ and $b / a-1$ with respect to pressure is due to the fact that the former is inversely proportional to the nearly vanishing shear constant $c_{s}$, whereas the latter depends on $c_{44}$ which is regular in this pressure range [see Eq. (2)].

In Fig. 3 we compare the enthalpy of the new orthorhombic phase (relative to that of the cubic phase) with that of the tetragonal phase previously conjectured to occur at higher pressures. The orthorhombic phase turns out to be more stable all over the explored pressure range.

In Fig. 4 we display our pressure versus volume data calculated for three different structures (cubic, tetragonal, and orthorhombic) and compare them with the equations of state fitted to experimental data from Refs. [2,8]. Our data are somewhat intermediate with respect to the previous equations of state, displaying a consistent decrease of the pressure when passing from the cubic to the tetragonal and orthorhombic phases.

We conclude with a brief comment on the effects of structural distortions on the optical properties. The gaps calculated for the tetragonal, cubic, and orthorhombic phases would close at around $V / V_{0} \approx 0.54,0.52$, and
0.51 , corresponding to pressures of $\approx 45,53$, and 55 $\mathrm{GPa}$, respectively. For a given molar volume, the tetragonal distortion tends to lower the gap [6], while the orthorhombic one increases it. It is well known that the LDA underestimates the optical gaps and we believe that the metallization pressure calculated using this approximation is (possibly slightly) underestimated.

This work has been partially supported by the Italian Consiglio Nazionale delle Ricerche under Grant No. 91.00943.69PF and by the European Research Office of the U.S. Army under Grant No. DAJA 45-89-C-0025.

[1] T.-L. Huang and A. Ruoff, Phys. Rev. B 29, 1112 (1984); T.-L. Huang, K. E. Brister, and A. L. Ruoff, Phys. Rev. B 30, 2968 (1984); Y. K. Vohra, K. E. Brister, S. T. Weir, S. J. Duclos, and A. L. Ruoff, Science 231, 1136 (1986).

[2] K. Asaumi, Phys. Rev. B 29, 1118 (1984).

[3] E. Knittle and R. Jeanloz, Science 223, 53 (1984); E. Knittle and R. Jeanloz, J. Phys. Chem. Solids 46, 1179 (1985).

[4] Y. K. Vohra, S. T. Weir, K. E. Brister, and A. Ruoff, Phys. Rev. Lett. 55, 977 (1985).

[5] N. E. Christensen and S. Satpathy, Phys. Rev. Lett. 55, 600 (1985).

[6] S. Baroni and P. Giannozzi, Phys. Rev. B 35, 765 (1987).

[7] S. Baroni and P. Giannozzi (unpublished).

[8] H. K. Mao, Y. Wu, R. J. Hemley, L. C. Chen, J. F. Shu, and L. W. Finger, Science 246, 649 (1989); H. K. Mao, Y. Wu, R. J. Hemley, L. C. Chen, J. F. Shu, L. W. Finger, and D. E. Cox, Phys. Rev. Lett. 64, 1749 (1990).

[9] I. V. Aleksandrov, A. F. Goncharov, I. N. Makarenko, and S. M. Stishov, Phys. Rev. B 43, 6194 (1991).

[10] P. W. Anderson and E. I. Blount, Phys. Rev. Lett. 14, 217 (1965).

[11] S. Baroni, P. Giannozzi, and A. Testa, Phys. Rev. Lett. 58, 1861 (1987); P. Giannozzi, S. de Gironcoli, P. Pavone, and S. Baroni, Phys. Rev. B 43, 7231 (1990).

[12] W. Bührer and W. Hälg, Phys. Status Solidi 46, 679 (1971).

[13] R. P. Lowndes, Phys. Rev. B 1, 2754 (1970).

[14] What we actually mean here by "phonon normal coordinates" are the eigenvectors of the matrix of the interatomic force constants. This definition would coincide with the usual one when the phonon frequency vanishes, or when the atomic masses are equal.

[15] L. D. Landau and E. M. Lifshitz, Statistical Physics (Pergamon, Oxford, 1980), Pt. I, Chap. XIV.

[16] J. S. Kim, D. M. Hatch, and H. T. Stokes, Phys. Rev. B 33, 1774 (1986).

[17] S. Baroni, P. Giannozzi, and M. Buongiorno Nardelli (unpublished). 


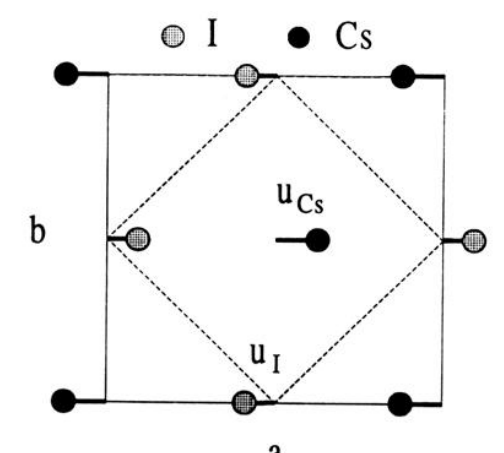

a

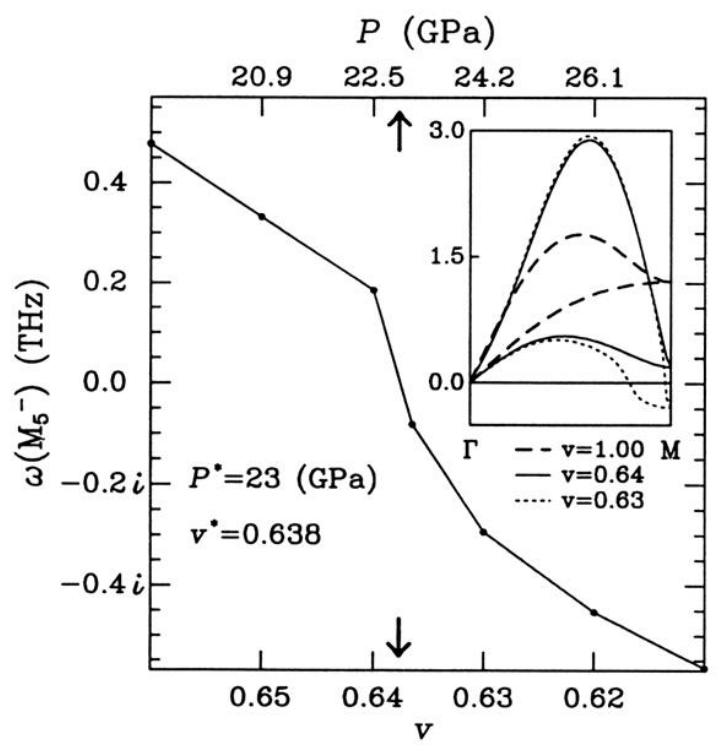

FIG. 1. Upper panel: Atomic displacements corresponding to an $M_{5}^{-}$acoustic phonon, viewed on the (001) plane. The magnitude of iodine displacements $\mathbf{u}_{\mathbf{I}}$ with respect to cesium displacements $\mathbf{u}_{\mathrm{Cs}}$ is exaggerated for clarity. The solid (dashed) line indicates the unit cell of the orthorhombic (cubic) structure. Iodine atoms lie on a plane shifted along the $z$ direction by $c / 2$ with respect to the cesium plane. In the cubic structure one has $a=b=\sqrt{2} c$. Lower panel: Frequency of the $M_{5}^{-}$phonon as a function of molar volume (lower scale, $v \equiv V / V_{0}$, where $V_{0}$ is the equilibrium volume) and of the pressure (upper scale). Arrows indicate the softening volume $\left(v^{*}\right)$ and pressure $\left(P^{*}\right)$. Inset: The lower portion of the acoustic dispersion along (110) at equilibrium volume (dashed line), and at volumes slightly above (solid line) and below (dotted line) the mode softening. 